\title{
Griffiths phase in the thermal quantum Hall effect
}

\author{
A. Mildenberger, ${ }^{1}$ F. Evers, ${ }^{1}$ R. Narayanan, ${ }^{1}$ A. D. Mirlin,,${ }^{1,2, *}$ and K. Damle ${ }^{3, \dagger}$ \\ ${ }^{1}$ Institut für Nanotechnologie, Forschungszentrum Karlsruhe, 76021 Karlsruhe, Germany \\ ${ }^{2}$ Institut für Theorie der Kondens. Materie, Universität Karlsruhe, 76128 Karlsruhe, Germany \\ ${ }^{3}$ Department of Theoretical Physics, Tata Institute of Fundamental Research, Mumbai 400005, India
}

(Received 1 April 2005; revised manuscript received 9 December 2005; published 2 March 2006)

\begin{abstract}
Two-dimensional disordered superconductors with broken spin-rotation and time-reversal invariance, e.g., with $p_{x}+i p_{y}$ pairing, can exhibit plateaus in the thermal Hall coefficient (the thermal quantum Hall effect). Our numerical simulations show that the Hall insulating regions of the phase diagram can support a sub-phase where the quasiparticle density of states is divergent at zero energy, $\rho(E) \sim|E|^{1 / z-1}$, with a nonuniversal exponent $z>1$, due to the effects of rare configurations of disorder (Griffiths phase).
\end{abstract}

DOI: 10.1103/PhysRevB.73.121301

PACS number(s): 73.43-f, 73.20.Fz, 75.10.Nr

The integer quantum Hall effect (IQHE) is observed in two-dimensional (2D) electron gases in high magnetic fields. Its striking manifestation is the quantization of the Hall conductance $\sigma_{x y}$ in units of $e^{2} / h$. Analogs of the IQHE are also known in systems with broken time reversal symmetry in which quasiparticle charge conservation is violated because of the presence of a charge condensate, while quasiparticle spin and energy remain conserved. In such a situation the Hall coefficients for spin or heat transport can exhibit plateaus at integer values in units of $\hbar / 2 \pi$ (Ref. 1) or $\pi k_{B}^{2} / 3 \hbar,{ }^{2,3}$ respectively. There has been a great deal of recent interest in these spin and thermal quantum Hall effects (SQHE and TQHE). ${ }^{1-8}$ In particular, the TQHE (Ref. 9) that one obtains in the absence of spin conservation displays many peculiar features that are not found in the IQHE or SQHE. ${ }^{3,11}$ These unusual properties arise in systems with paired fermions without spin conservation, which belong to class D in the Altland-Zirnbauer scheme. ${ }^{10}$ These systems find a natural realization in disordered superconductors with triplet ( $p$-wave) pairing, e.g., $\mathrm{Sr}_{2} \mathrm{RuO}_{4}{ }^{12,13}$

Another candidate pertinent for class D physics is the $\nu$ $=5 / 2$-state in the fractional quantum Hall effect, that exhibits pairing of composite fermions. ${ }^{14-16}$ This state is observed almost routinely in experiments. ${ }^{17,18}$ According to recent proposals, this state can be used for quantum computing, ${ }^{19}$ as its localized zero energy excitations (zero modes) exhibit nonAbelian statistics. ${ }^{14}$

In this work, we investigate the effect of disorder on the density of states (DOS) of such 2D systems in symmetry class D, choosing as a model system a Chalker-Coddingtontype network, which is a fermionic representation of the $\pm J$ random bond Ising model (RBIM). Our choice is motivated by the fact that much is known about this system, including the location of the phase boundary ${ }^{20}$ separating the ferro- and paramagnetic phases (which in the fermionic language translates into a transition between two insulating Hall phases). The two phases are distinguished from each other via the value of their dimensionless Hall conductance, see Ref. 3. The quantum Hall transition between these two insulating phases has been extensively studied. ${ }^{20}$ As we discuss at the end of the paper, our results are however not restricted to this model but rather are generally valid for TQHE systems.

Recently, Gruzberg et al. ${ }^{21}$ suggested that the RBIM has insulating phases supporting a region where the DOS exhibits a power-law singularity $\rho(E) \propto|E|^{-1+1 / z}$ with a nonuniversal exponent $z$. Strong-disorder renormalization group (RG) calculations of Motrunich et al. ${ }^{22}$ have also demonstrated the presence of such a divergence in the DOS (with $z>1$ ) in quasi-one-dimensional (1D) superconductors with broken time reversal and spin rotation invariance. This has also been confirmed by calculations using a Fokker-Planck approach. ${ }^{23}$ The strong-disorder RG calculations make it apparent that the DOS divergence originates in this and in other 1D models ${ }^{24}$ from Griffiths effects ${ }^{25}$ involving exponentially rare length $\ell$ regions (occurring with probability $\sim e^{-c^{\prime} \ell}$ ) of the sample in nearby phases. An exponentially weak coupling, $\sim e^{-c \ell}$, of defects at the boundaries of such rare regions produces a power-law tail in the low-energy DOS:

$$
\rho(E) \sim \int d \ell \delta\left(E-e^{-c \ell}\right) e^{-c^{\prime} \ell} \propto|E|^{1 / z-1}
$$

$\left(z=c / c^{\prime}\right.$ and $c, c^{\prime}$ are nonuniversal parameters).

Gruzberg et al. ${ }^{21}$ and Motrunich et al. ${ }^{22,29}$ further conjectured that a similar mechanism drives a divergent DOS also in 2D systems in class D. We stress that this extension of 1D physics to a generic system in two dimensions is far from obvious, and we provide here the first detailed evidence and understanding of the same.

A regime with a divergent DOS is in sharp contrast to the situation in standard Wigner-Dyson ensembles, where the DOS is never divergent. In 2D power-law divergences, generically modified by logarithmic corrections (the Gade form of the density of states ${ }^{27,29}$ ) have been known to exist in very special systems belonging to the chiral (or particle-hole symmetric) symmetry classes ${ }^{26,28}$ that are very difficult to realize experimentally. This Gade form of the DOS arises in critical phases without exponential localization of the wave functions. Pure power laws are only known to occur under highly contrived circumstances, namely in chiral systems with probability distributions of random couplings that are not translationally invariant. ${ }^{29}$ By contrast, here we report an observation of an insulating Griffiths phase with power-law singularities in the DOS of a model (RBIM) in the TQHE universality class with full (statistical) translational invari- 

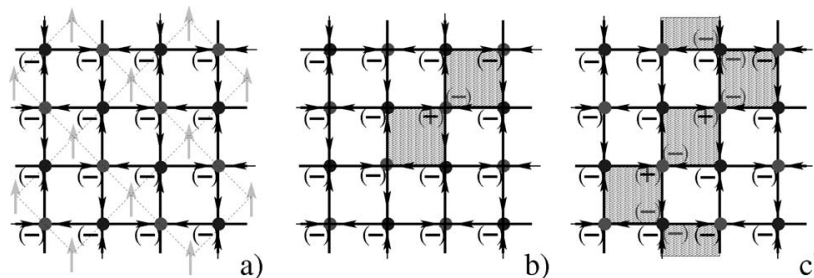

FIG. 1. 2D Ising models in a representation of fermions moving on a chiral network: (a) Clean system; spin sites of the Ising model in its original spin representation are also shown, (b) network carrying an isolated vortex (hatched plaquettes). Vortex pairs are realized by inserting two half integer flux quanta into a pair of plaquettes sharing a single node, and (c) a string of four vortex pairs.

ance. We further demonstrate numerically that the divergent DOS is indeed governed by a $1 \mathrm{D}$ mechanism involving the presence of rare long string defects. Our numerical results thus show that TQHE-in contrast to its close relatives, the IQHE and the SQHE_-does exhibit the conjectured Griffiths phase. $^{21,22}$

The RBIM network that we use for our calculations is a square lattice comprised of directed links [Fig. 1(a)]. A state of the network is defined by assigning a complex (probability) amplitude to every link. The state's evolution in a single time step is governed by an operator $U$. It generates a probability flow that follows the link's direction and turns left or right with probability $t_{l}$ and $1-t_{l}$ at a node. Consistent with class D symmetry, the flow along the network links picks up a phase factor of $\pi$ on winding around the plaquette once, hence the $(-)$ signs. Disorder (realizing antiferromagnetic couplings, $J \rightarrow-J$ ) is introduced by vortex pairs that are attached to the network nodes, see Fig. 1(b).

The network is characterized by two parameters: the concentration of vortex pairs, $p$, and the inter-plaquette coupling, $t_{l}=\sin ^{2} \alpha$. The latter can be related to the temperature $T$ and coupling constant $J$ of the Ising model formulated in terms of lattice spins via $t_{l}=\cosh ^{-2} 2 J / T$. At low $T$, the fermions wind preferably about plaquettes not carrying spins: $t_{l} \approx 0$. Figure 2 shows the model's phase diagram. The phases of the spin system-ferro- and paramagnetic-correspond to two insulating phases of the network fermions. The insulating character is easily understood in the clean case, where it is a consequence of band structure: In the two limiting cases $t_{l}$ $=0$ and $t_{l}=1$ fermions wind about their own plaquettes indefinitely. Then the energy spectrum is degenerate, and the Fermi energy $E=0$ lies in a gap. Only at $t_{l}=1 / 2$, i.e., on the transition line, the gap closes. The gap is robust against introducing a weak concentration of antiferromagnetic bonds ${ }^{30,31}$ and therefore two insulating phases develop.

We construct $U$ for a single time step for networks with periodic boundary conditions according to the prescription implied by Fig. 1. Its eigenvalues come in quadruplets, $\pm e^{ \pm i E}$ because $U$ is unitary, real and obeys a chiral sublattice symmetry: Hopping is allowed only from one sublattice to the other. In the limit $t_{l}=0$, the eigenvalues are most easily obtained by noting that $e^{i 4 E}=-1$ when the fermion moves about a clean plaquette and $e^{i 4 E}=1$ if the plaquette is pierced by a vortex. In the latter, $E=0$ is a valid solution. These are the zero modes mentioned in the introduction.

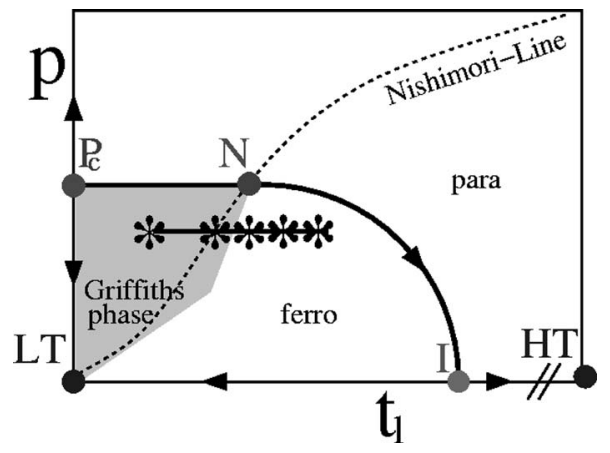

FIG. 2. Phase diagram of TQHE in RBIM-network model representation, disorder $p$ vs interplaquette coupling strength (temperature) $t_{l}$. Fixed points: High temperature (HT), low temperature (LT), clean Ising $(\mathrm{I})$, multicritical point $(\mathrm{N})$, and percolation $\left(\mathrm{P}_{\mathrm{c}}\right)$. Phase boundary (solid, precise location calculated in Ref. 20) separates two Hall insulators (called ferro and para using RBIM terminology). The Nishimori line: a line which supports a local $Z_{2}$ symmetry and where the internal energy of the model is analytic. Shaded region indicates Griffiths phase with diverging single particle density of states, $\rho(E)$; in plain white regions DOS has (pseudo)-gap. Line segments represent scans for $\rho(E)$ displayed in Fig. 3. In Fig. 4 , scan is along phase boundary from $\mathrm{N}$ to $\mathrm{P}_{\mathrm{c}}$.

In order to extract the eigenvalues closest to zero at $t_{l}>0$, we use standard sparse matrix packages. ${ }^{32}$ Typically 8 to 16 eigenvalues per system are calculated. We obtain the density of (pseudo)-energies, $\rho(E)$, from an average over an ensemble of disorder realizations which contains typically on the order of $10^{4}$ members.

As expected, we find from this procedure that at low concentration of disorder (and actually in the entire paramagnetic phase) the gap of the clean system survives (see Fig. 3). More precisely, due to the Lifshitz mechanism it turns into a pseudo-gap. ${ }^{33}$ However, if we start in the ferromagnet at large values of the interplaquette coupling strength $t_{l}$ and diminish it at fixed disorder so as to get close to the Nishimori line (NL), we witness a qualitative change in the lowenergy DOS: The pseudo-gap vanishes and the DOS starts to increase with lowering $E$, apparently diverging as $E \rightarrow 0$. This is demonstrated in Fig. 3, where the evolution of DOS is shown as $t_{l}$ is lowered from 0.13 down to 0.014 . For large enough $t_{l}$, one still notices the pseudo-gap behavior. The latter is already less evident in the data that corresponds to the coupling strength $t_{l}=0.07$. It finally gives way to a strongly increasing behavior once $t_{l}$ falls below $\approx 0.05$.

Having demonstrated the DOS divergence in the low- $T$ part of the ferromagnetic phase, we now discuss the corresponding mechanism. We will show that the singularity is governed by rare disorder configurations containing long chains (strings) of defects. Since each defect contains a pair of vortices, such a string of a length $\ell>1$ (formed with a probability $\sim p^{\ell}$ ) produces effectively two vortices with a large separation $\sim \ell$.

At $t_{l}=0$, every vortex in the system contributes a zero mode to the spectrum, which therefore has a macroscopic degeneracy $\sim p L^{2}$ at $E=0$. Upon switching on the coupling $t_{l}$, the zero modes couple and the degeneracy is lifted. For a small $t_{l}$, the energy splitting $E$ thus produced will be expo- 


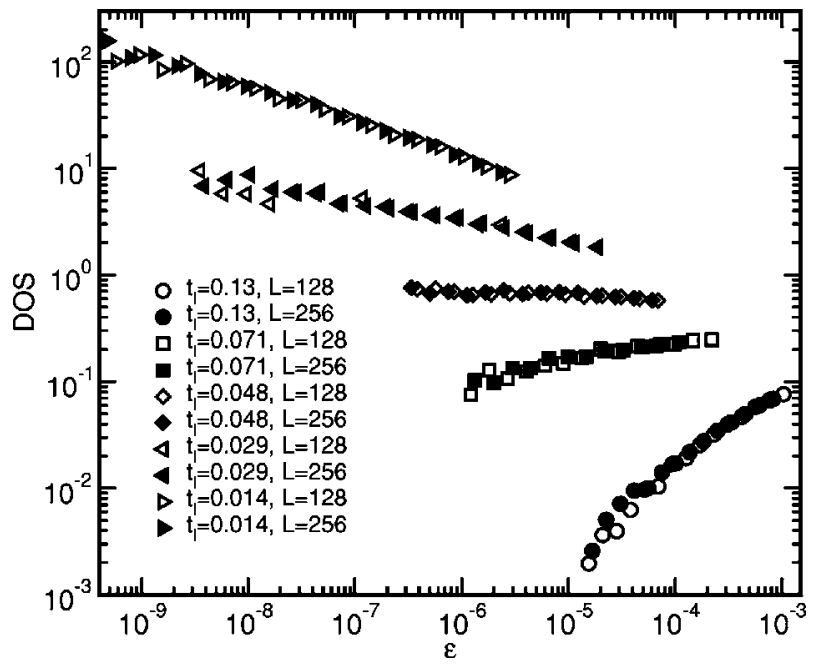

FIG. 3. DOS at disorder value $p=0.08$ deep in the ferromagnetic phase. As coupling between plaquettes is progressively reduced, pseudo-gap gives way to power law divergency. Plots show averages for two system sizes, 128 (256) with 20,000 $(10,000)$ realizations. Data comprise 8 lowest lying eigenstates; $t_{l}=0.029$ corresponds to location on Nishimori line.

nentially small in $\ell{ }^{30}$ For a clean system (which contains only two vortices at the ends of the string but no other defects) this follows immediately from the perturbation theory, yielding $E \propto t_{l}^{\ell / 2}=e^{-c \ell}$. Clearly, the same result holds for those rare configurations of the disordered system for which the region of a size $\sim \ell$ around the vortex pair is free of other vortices. However, the probability for this to happen scales with $\ell$ as $(1-p)^{\ell^{d}}$, where $d=2$ is the spatial dimensionality. In the $1 \mathrm{D}$ case this factor is harmless, yielding the probability for formation of an isolated string $\propto e^{-c^{\prime} \ell}$ with $c^{\prime}=-\ln p(1-p)^{2}$ and Eq. (1) applies. On the other hand, in $d=2$, the above factor seems to spoil the reasoning leading to Eq. (1). So, if the requirement of isolation of the vortex pair would be a necessary condition for the appearance of exponentially small eigenvalues, $\epsilon \sim e^{-c \ell}$, the divergent DOS would be converted into a pseudo-gap at lowest energies due to the $(1-p)^{\ell^{d}}$ factor.

We now argue that this requirement is not necessary, and Eq. (1) is applicable in two dimensions as well. Indeed, at small $p$ almost all randomly located defects will be isolated. Correspondingly, $\simeq p$ is the concentration of vortex pairs with separation unity and an energy splitting $\sim t_{l}^{1 / 2}$. There will be a much smaller concentration $\left(p^{2}\right)$ of defects forming chains of length two, yielding the energy splitting $\sim t_{l}$, and so forth. The energy splitting associated with these nearby (compared to $\ell$ ) vortices will be relatively big, and will only weakly affect the energy splitting $t^{\ell / 2}$ of the $\ell$-vortex. This argument can be converted to a RG procedure, as in Refs. 22 and 29. Vortices can be sorted into pairs by combining those with the shortest distance first, the second shortest distance next, etc. Since the energy splitting associated with the coupling of two vortices is exponential in their distance, pairs differing in this distance will strongly differ in their splitting as well. The hierarchical order allows for a RG procedure that successively eliminates vortex pairs with an energy split-

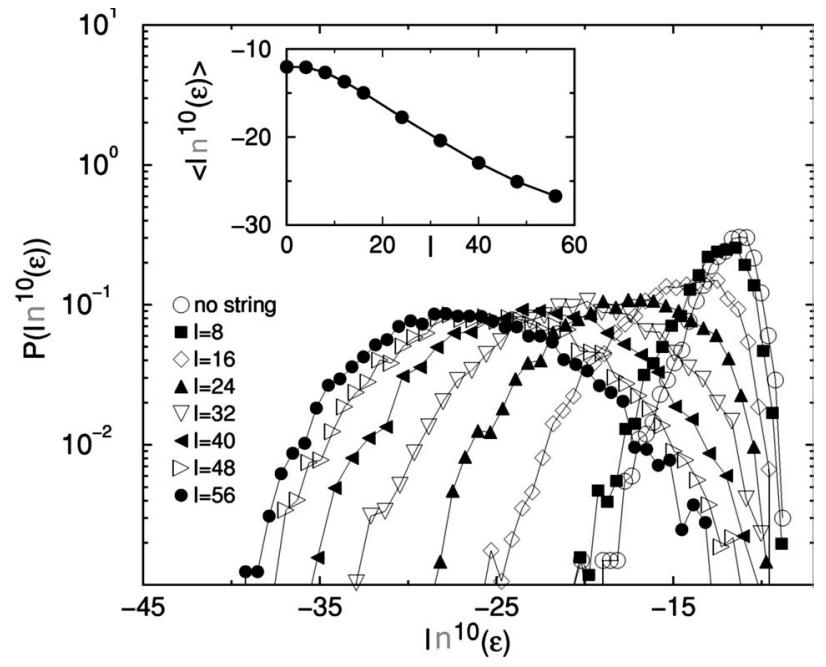

FIG. 4. Distribution of logarithm of lowest eigenvalue for string of defects of length $\ell=8-56$ inserted in disordered system in ferromagnetic phase $\left(p=0.08, t_{l}=0.029, L=128\right)$. Inset: Typical lowest eigenvalue as a function of $\ell$.

ting that is large as compared to the pairs remaining on the lattice. Clearly, for sufficiently small $t_{l}$ and $p$ (the boundary of the Griffiths phase will be discussed below) the eigenvalues associated with long strings, i.e., vortex pairs with a large separation, are (largely) insensitive to the presence of other vortex pairs. In other words, an $\ell$-string does not have to be isolated in order to contribute an eigenvalue $\sim e^{-c \ell}$. The only role of other (randomly distributed) vortices will be in somewhat modifying the parameter $c$ as compared to its bare value $c=-1 / 2 \ln t_{l}$.

The string mechanism, which is crucial for the proposed $\mathrm{RG}$ argument, has been validated by a numerical test. We have inserted a string of the length $\ell(\ell=8-56)$ into a disordered network with $t_{l}=0.029$ and $p=0.08$. Figure 4 displays the evolution of the distribution of the lowest eigenvalue with increasing length of the string. The results fully confirm our scenario: (a) The extra string gives rise to an additional, isolated eigenvalue; (b) this eigenvalue moves with increasing $l$ according to the expected exponential law, $\langle-\ln \epsilon\rangle \propto \ell$, see inset of Fig. 4 (the rest of the spectrum does not move). We have performed the same calculation in the paramagnetic phase $\left(p=0.2, t_{l}=0.029\right)$ and found that the distribution does not move with $\ell$ : A long string of defects in a random environment does not generate an exponentially small eigenvalue.

Therefore, the analytical arguments together with the numerical results, Figs. 3 and 4, demonstrate the existence of a Griffiths phase where string formation generates a divergent DOS, Eq. (1) with $z>1$. This phase is shaded in the phase diagram, Fig. 2. Its boundary on the side of larger $t_{l}$ is given by the condition $z=1$ and, according to our numerical results, is at least close to the NL. The asymptotic behavior of the boundary at low $T$ and weak disorder can be easily found from our analytical considerations. Indeed, in this limit $c \simeq-1 / 2 \ln t_{\ell} \simeq 2 J / T$, and $c^{\prime}=-\ln p$, so that the condition $z$ $=c / c^{\prime}=1$ reduces to $\ln p \simeq-2 J / T$. This agrees with the low$T$ asymptotics of the NL, whose exact form is $e^{2 J / T}=p /(1$ 
$-p$ ). It is thus plausible that the boundary of the Griffiths phase with divergent DOS in fact coincides with the NL as was conjectured in Ref. 21.

The boundary at large $p$ is the phase transition line into the paramagnet, where the string mechanism ceases to be operative. Right here, between the Nishimori point $N$ and the percolation critical point $P_{c}$, we find a divergent DOS with a nonuniversal exponent $z$. While we do not have at present an analytical theory for this behavior of DOS at criticality, an analogy with the random phase $X Y$ model, which has a phase diagram remarkably similar to Fig. 2 , might be instructive. ${ }^{34}$

In conclusion, we have demonstrated that a type of insulating phase for 2D electron systems characterized by a diverging DOS occurs in the TQHE. We have shown that this
Griffiths-type singularity is related to the formation of strings of defects leading to an exponentially small splitting of zero modes. Our numerical work is based on the network representation of the $\pm J$ random bond Ising model. Preliminary results on the more generic Cho-Fisher model ${ }^{35}$ suggest that the Griffiths phase is a ubiquitous companion of the TQHE.

The authors acknowledge valuable discussions with D. Beckmann, J. T. Chalker, I. A. Gruzberg, A. W. W. Ludwig, C. Mudry, X. Wan, and P. Wölfle and support by the DFG under SPP "Quanten-Hall-Systeme" and by the Sloan Foundation for one of the authors (K.D.). That author (K.D.) would also like to thank D. S. Fisher for generously sharing his unpublished preliminary results.
*Also at Petersburg Nuclear Physics Institute, 188350 St. Petersburg, Russia.

†Previously at: Department of Physics and Astronomy, Rice University, Houston, TX.

${ }^{1}$ T. Senthil, J. B. Marston, and M. P. A. Fisher, Phys. Rev. B 60, 4245 (1999).

${ }^{2}$ A. Vishwanath, Phys. Rev. Lett. 87, 217004 (2001).

${ }^{3}$ T. Senthil and M. P. A. Fisher, Phys. Rev. B 61, 9690 (2000).

${ }^{4}$ R. Bundschuh, C. Cassanello, D. Serban, and M. R. Zirnbauer, Phys. Rev. B 59, 4382 (1999).

${ }^{5}$ I. A. Gruzberg, A. W. W. Ludwig, and N. Read, Phys. Rev. Lett. 82, 4524 (1999).

${ }^{6}$ E. J. Beamond, J. Cardy, and J. T. Chalker, Phys. Rev. B 65, 214301 (2002).

${ }^{7}$ V. Kagalovsky, B. Horovitz, Y. Avishai, and J. T. Chalker, Phys. Rev. Lett. 82, 3516 (1999).

${ }^{8}$ A. D. Mirlin, F. Evers, and A. Mildenberger, J. Phys. A 36, 3255 (2003); F. Evers, A. Mildenberger, and A. D. Mirlin, Phys. Rev. B 67, 041303(R) (2003).

${ }^{9}$ J. T. Chalker, N. Read, V. Kagalovsky, B. Horovitz, Y. Avishai, and A. W. W. Ludwig, Phys. Rev. B 65, 012506 (2001).

${ }^{10}$ A. Altland and M. R. Zirnbauer, Phys. Rev. B 55, 1142 (1997); M. R. Zirnbauer, J. Math. Phys. 37, 4986 (1996).

${ }^{11}$ M. Bocquet, D. Serban, and M. R. Zirnbauer, Nucl. Phys. B 578, 628 (2000).

${ }^{12}$ K. D. Nelson, Z. Q. Mao, Y. Maeno, and Y. Liu, Science 306, 1151 (2004).

${ }^{13}$ For a review see, e.g., P. J. Hirschfeld and W. A. Atkinson, J. Low Temp. Phys. 126, 881 (2002); A. Altland, B. D. Simons, and M. R. Zirnbauer, Phys. Rep. 359, 283 (2002).

${ }^{14}$ G. Moore and N. Read, Nucl. Phys. B 360, 362 (1991).

${ }^{15}$ R. H. Morf, Phys. Rev. Lett. 80, 1505 (1998).

${ }^{16}$ N. Read, Physica B 298, 121 (2001).
${ }^{17}$ J. P. Eisenstein, K. B. Cooper, L. N. Pfeiffer, and K. W. West, Phys. Rev. Lett. 88, 076801 (2002).

${ }^{18}$ J. S. Xia, W. Pan, C. L. Vicente, E. D. Adams, N. S. Sullivan, H. L. Stormer, D. C. Tsui, L. N. Pfeiffer, K. W. Baldwin, and K. W. West, Phys. Rev. Lett. 93, 176809 (2004).

${ }^{19}$ S. DasSarma, M. Freedman, and C. Nayak, Phys. Rev. Lett. 94, $166802(2005)$

${ }^{20}$ F. Merz and J. T. Chalker, Phys. Rev. B 65, 054425 (2002).

${ }^{21}$ I. A. Gruzberg, N. Read, and A. W. W. Ludwig, Phys. Rev. B 63, 104422 (2001).

${ }^{22}$ O. Motrunich, K. Damle, and D. A. Huse, Phys. Rev. B 63, 224204 (2001)

${ }^{23}$ I. A. Gruzberg, N. Read, and S. Vishveshwara, cond-mat/ 0412413.

${ }^{24}$ K. Damle, Phys. Rev. B 66, 104425 (2002).

${ }^{25}$ R. B. Griffiths, Phys. Rev. Lett. 23, 17 (1969).

${ }^{26}$ A. W. W. Ludwig, M. P. A. Fisher, R. Shankar, and G. Grinstein, Phys. Rev. B 50, 7526 (1994).

${ }^{27}$ R. Gade, Nucl. Phys. B 398, 499 (1993); F. Wegner and R. Gade, ibid. 360, 213 (1991).

${ }^{28}$ C. Mudry, S. Ryu, and A. Furusaki, Phys. Rev. B 67, 064202 (2003).

${ }^{29}$ O. Motrunich, K. Damle, and D. A. Huse, Phys. Rev. B 65, 064206 (2002).

${ }^{30}$ N. Read and A. W. W. Ludwig, Phys. Rev. B 63, 024404 (2000).

${ }^{31}$ A. Mildenberger and F. Evers (unpublished).

${ }^{32}$ J. W. Demmel et al., SIAM J. Matrix Anal. Appl. 20, 720 (1999); R. B. Lehoucq, D. Sorensen, and C. Yang, ARPACK Users Guide (SIAM, Philadelphia, 1998).

${ }^{33}$ I. M. Lifshitz, Sov. Phys. JETP 17, 1159 (1963).

${ }^{34}$ D. Carpentier and P. Le Doussal, Nucl. Phys. B 588, 565 (2000).

${ }^{35}$ S. Cho and M. P. A. Fisher, Phys. Rev. B 55, 1025 (1997). 